\title{
WILEY
}

\section{Preface to the Special Issue on Matheuristics and Metaheuristics}

This special issue of the International Transactions in Operational Research focuses on Matheuristics and Metaheuristics and is the largest published to date, highlighting the importance of the field and the broad scope of these methods and the reach of their applications.

Academicians and practitioners responded with enthusiasm to three parallel calls for papers, each of them focusing on a different theme, but all related to approximate methods to solve optimization problems: Matheuristics, Metaheuristics, and Variable Neighborhood Search. More than 120 articles were submitted and handled by the Guest Editors.

After several rounds of refereeing and the conclusion of the peer review procedure, the 28 accepted articles appear in this special issue. In order to make it clear that they are all correlated and to better illustrate the cross-fertilization between the themes of this special issue, these articles appear in the alphabetical order of the last name of their first authors, and not in separate clusters. Many (or most) articles in this issue could certainly fit in more than one of the themes that motivated the three original calls for papers.

I would like to express my gratitude to the Guest Editors of this special issue - who are named here in alphabetical order: Christian Blum, Angel Juan, Vittorio Maniezzo, Nenad Mladenović, Helena Ramalhinho, Angelo Sifaleras, Kenneth Sörensen, Marcone Souza, and Thomas Stützle_-for their support and their commitment to this project. I would like also to join the Guest Editors to acknowledge the hard work put in by the referees in the revision of all submitted papers. Last, but certainly not least, all Editors would like to thank the Authors for their contribution to this special issue, which would not exist without their high quality work. We sincerely believe that the readers will enjoy reading these articles.

Celso C. Ribeiro

General Editor

\section{Short preface - Matheuristics}

Matheuristics are methods that exploit mathematical programming techniques in heuristic and metaheuristic frameworks, granting to mathematical programming approaches the problem robustness and time effectiveness that characterize heuristics, or exploiting the mathematical programming 
model formulation in the customization of a heuristic for some specific problem. In fact, progress in mathematical programming has led to a state of the art where exact methods, most notably mixed integer programming solvers, can be effective even in a heuristic context, both as primary solvers or as sub-procedures.

Matheuristics have become an important complement of exact approaches in optimization research. Admittedly, mathematical programming has been used in the design of heuristics ever since its beginning and heuristic algorithms with mathematical programming components have often been simply referred to as "hybrids", with no reference to matheuristics. However, the availability of a signifier to denote a signified is very helpful. It helps to conceptualize the area, which is no longer seen as a scattered array of diverse contributions, as well as in discussions and confrontations. Thus, ultimately, it helps research.

We are now aware of matheuristic-specific approaches, such as the corridor method or kernel methods, not to mention Very Large-Scale Neighborhood Search (VLSNS) or local branching, as well as of matheuristic-specific contributions to established approaches, such as to genetic algorithms or Greedy Randomized Adaptive Search Procedure (GRASP). There are established matheuristic research lines, while innovative approaches are presented and appreciated as such. There are dedicated forums to the presentation of specific matheuristic results. The matheuristic articles that appear in this special issue are extended versions of conference presentations originally proposed in one of the workshops of the Matheuristics series, which is one of the main outlets of the area.

In fact, this special issue features contributions that originally appeared in the 2016 Matheuristics workshop. This was the sixth workshop of a series initiated in 2006 that regularly continued with one event every two years. The first two workshops were organized in Bertinoro, Italy, in 2006 and 2008. The third took place in Vienna, Austria, in 2010. The series left Europe for the fourth workshop, which was held in Angra dos Reis, Brazil, in 2012. Back to Europe, the following workshops took place in Hamburg, Germany, in 2014 and, finally, in Brussels, Belgium, in 2016. Overall, more than 300 researchers attended at least one of the six workshops in the series, tackling a wide scope of problems and presenting a broad range of methodologies, all sharing the common design approach of exploiting the mathematical structure of the problem of interest to get to an effective heuristic for it. The Matheuristics 2016 workshop that motivated the call for papers that attracted the papers appearing in this special issue was organized at the Universite libre de Bruxelles and featured more than 20 presentations and three invited lectures, showcasing the varied and intrinsically nontrivial nature of the research in this area.

Vittorio Maniezzo

Università di Bologna, Italy

Thomas Stützle

Université libre de Bruxelles, Belgium

\section{Short preface - Metaheuristics}

This special issue also includes high-quality articles presented at the 12th Metaheuristics International Conference (MIC 2017), which was held in Barcelona, Spain, from July 4 to 7, 2017. 
Established in 1995, the MIC series of international conferences is one of the main events focusing on the progress of metaheuristics and their applications to different fields: from transportation and logistics, to telecommunication systems, and computational finance. As in the previous editions, MIC 2017 provided an opportunity for the international research community in the area of metaheuristics to gather and discuss recent research results, to develop new ideas, and to generate new cooperation links in a friendly and relaxed atmosphere.

MIC 2017 enjoyed around 250 participants and featured about 170 talks. The presentations at MIC 2017 covered several aspects of the research on metaheuristics, such as new algorithmic developments, industrial applications, new research challenges, theoretical developments, implementation issues, in-depth experimental studies, and hybrid metaheuristics.

Apart from the metaheuristics themselves, presentations at MIC 2017 also dealt with techniques that enhance the usability - and increase the potential — of metaheuristics, such as the parallelization of algorithms, reactive search mechanisms for self-tuning, offline metaheuristic algorithm configuration techniques, and algorithm portfolios.

Empirical and theoretical research in metaheuristics included large-scale experimental analyses, algorithm comparisons, new experimental methodologies, engineering methodologies for stochastic local search algorithms, search space analysis, and theoretical insights into properties of metaheuristics. Among the main problems considered, we found well-known and classical ones such as the traveling salesman problem, vehicle routing, scheduling, location, and matching.

One of the most appealing aspects of metaheuristics is their potential applicability in the solution of industrial problems in many different sectors, such as transportation, health care, bioinformatics, data mining, planning and scheduling, production and operations management, economics, marketing, telecommunications, logistics, and supply chain management. New innovative applications of metaheuristics in industry arise on an almost daily basis, thus leading to a high impact on organizational performance.

Finally, we would like to mention the importance of the interface of metaheuristics with other disciplines, such as agent-based models, integer programming, constraint programming, machine learning, and deep learning. Likewise, the area incorporates new challenging problems related to big data and large-scale optimization problems, multi-objective, stochastic, and dynamic problems, as well as new and challenging problems in interdisciplinary fields such as sustainable smart cities.

This special issue features metaheuristic articles covering important real-world applications in health care, steel making, and the oil industry, as well as application-oriented variations of more classical problems such as the travelling salesman problem and the vehicle routing problem with time windows.

\author{
Christian Blum \\ Artificial Intelligence Research Institute (IIIA-CSIC), Spain \\ Angel A. Juan \\ Open University of Catalonia, Spain \\ Helena Ramalhinho \\ Universitat Pompeu Fabra, Spain \\ Thomas Stützle \\ Université libre de Bruxelles, Belgium \\ (C) 2019 The Authors.
} International Transactions in Operational Research (C) 2019 International Federation of Operational Research Societies 


\title{
Short preface - Variable Neighborhood Search
}

The aim of the papers featured in this theme of this special issue is to present new trends in Variable Neighborhood Search (VNS). This methodology, which was originally proposed by Nenad Mladenović and Pierre Hansen, is based on systematic changes in the neighborhood structure along the search. These changes can be performed within the mathematical programming formulation of the problem considered (matheuristics) or within combinatorial formulations (metaheuristics), making VNS suitable for a special issue devoted to matheuristics and metaheuristics. The VNS methodology has been successfully applied in the solution of various combinatorial and global optimization problems, and related tasks, in the framework of both matheuristics and metaheuristics.

The VNS papers in this special issue originated in two recent meetings of the International Conference on Variable Neighborhood Search (ICVNS) series: ICVNS'16 and ICVNS'17. This series of conferences is devoted to the use of the VNS approach in optimization.

The 4th International Conference on Variable Neighborhood Search (ICVNS'16) was held in Málaga, Spain, from October 3 to 5, 2016. It was attended by 60 participants and its program included 59 talks distributed across 14 contributed sessions during the three days. The 5th International Conference on Variable Neighborhood Search (ICVNS'17) was held in Ouro Preto, Brazil, from October 2 to 4, 2017. It was attended by 80 participants and the conference program included 32 talks distributed across nine contributed sessions, once again during the three days of the meeting.

The best papers of these two ICVNS conferences have been selected and submitted for this special issue. Each submission was peer reviewed by at least two referees, according to the editorial policy of the International Transactions in Operational Research.

\author{
Nenad Mladenović \\ Mathematical Institute, SANU, Serbia \\ Angelo Sifaleras \\ University of Macedonia, Greece \\ Kenneth Sörensen \\ University of Antwerp, Belgium \\ Marcone J.F. Souza \\ Universidade Federal de Ouro Preto, Brazil
}

(C) 2019 The Authors.

International Transactions in Operational Research (C) 2019 International Federation of Operational Research Societies 